\title{
Entwicklung der nationalen S3-Leitlinie „Diagnostik, Therapie und Nachsorge des Mammakarzinoms“
}

\author{
A. Wöckel, R. Kreienberg (Leitlinienkoordinator der S3-Leitlinie „Diagnostik, Therapie und Nachsorge des Mammakarzinoms“ \\ und 2. Vizepräsident der Deutschen Gesellschaft für Gynäkologie und Geburtshilfe)
}

Der Umfang des medizinischen Wissens vermehrt sich gerade in dynamischen Fachbereichen wie der Gynäkoonkologie und speziell beim Mammakarzinom exponentiell. In fast 30000 medizinischen Fachjournalen werden derzeit knapp 3 Millionen neue Artikel pro Jahr publiziert. Der rasante Zuwachs dieser Daten bedingt, dass die „Halbwertszeit“ des medizinischen Fachwissens schnell abnimmt. Parallel zu dieser Entwicklung entstehen zwar neue und moderne Informationstechnologien, welche jederzeit einen schnellen Zugriff auf Daten ermöglichen. Die methodisch hochwertigen und handlungsrelevanten Informationen können jedoch häufig nur schwer extrahiert werden. Klinische Leitlinien kombinieren den relevanten Extrakt dieses Wissenszuwachses mit der Erfahrung und Einschätzung renommierter Fachexperten, welche im Leitlinienerstellungsprozess beteiligt sind.

Bereits 2004 wurde daher die erste Version der deutschen S3Leitlinie zur Diagnostik, Therapie und Nachsorge des Mammakarzinoms nach den Vorgaben des Leitlinien-Manuals der deutschen Arbeitsgemeinschaft Wissenschaftlich Medizinischer Fachgesellschaften (AWMF) und des Ärztlichen Zentrums für Qualität in der Medizin (ÄZQ) unter der Federführung des Leitlinienkoordinators der DGGG Prof. Dr. Rolf Kreienberg veröffentlicht. Für die Erarbeitung der ersten Version wurde eine umfassende systematische Recherche, Auswahl und Bewertung hochwertiger Literatur für versorgungsrelevante Fragestellungen durchgeführt. Dabei wurden zunächst die Publikationen und Empfehlungen nach den Kriterien der evidenzbasierten Medizin klassifiziert, bevor eine Konsensusfindung mit den Fachexperten aller beteiligten Fachgesellschaften erzielt wurde.

Um explizite Handlungsempfehlungen nachvollziehbar darstellen zu können, wurden bereits zu diesem Zeitpunkt klinische Algorithmen entwickelt, um wesentliche und klinisch relevante Schritte in einfachen Flussdiagrammen darzustellen. Auch eine Formulierung von Qualitätszielen und -indikatoren mit Ziel- und Referenzbereichen für die Prozess- und Ergebnisqualität waren Inhalt der ersten deutschen S3-Leitlinie. Diese Indikatoren wurden bereits als Bestandteile des Qualitätsmanagements in das Zertifizierungssystem der Deutschen Krebsgesellschaft (DKG) und Deutschen Gesellschaft für Senologie (DGS) für die Zertifizierung von Brustzentren übernommen.

Leitlinien müssen in regelmäßigen Abständen aktualisiert werden. Durch den enormen Informationszuwachs können evidenzbasierte Leitlinien in der Regel zwei bis drei Jahre gültig sein und bedürfen dann einer Aufarbeitung mit den aktuellsten Studienergebnissen. Daher wurde für die S3-Leitlinie Mammakarzinom im Frühjahr 2006 das nötige Aktualisierungsverfahren eingeleitet. Die überarbeitete Version wurde im Februar 2008 publiziert. Herausgeber der Leitlinie war das Koordinierungszentrum für Standards in der Onkologie (ISTO) der Deutschen Krebsgesellschaft e.V. (DKG). Die DKG, die Deutsche Krebshilfe und die AWMF sind im „Onkologischen Leitlinienprogramm“ die federführenden Gesellschaften. Mitherausgeber sind weitere an dem Versorgungsproblem Mammakarzinom beteiligte Fachgesellschaften, Arbeitsgemeinschaften und Organisationen, die renommierte Experten für den Entwicklungsprozess nominiert haben. Der Abschluss einer zweiten Aktualisierung wird für 2011 erwartet.

Um international gültige methodische Anforderungen $\mathrm{zu}$ erfüllen, wird primär eine erneute systematische Literaturrecherche mit Auswahl der aktuellsten Veröffentlichungen durchgeführt. Die Recherche bezieht die medizinischen Datenbanken Medline, BIOSIS, Previews, CDSR, ACP Journal Club, DARE, CCTR, CINHAL ein. Der Suchzeitraum erstreckt sich über einen Zeitraum von 2006 bis in die Gegenwart. Durch entsprechende inhaltliche und methodische Selektion durch die Experten der beteiligten Fachgesellschaften werden die methodisch belastbaren Quellen identifiziert und als Evidenzgrundlage für die Überprüfung und Neuformulierung der Handlungsempfehlungen herangezogen. 
Die Leitlinie nutzt als Grundlage zur Evidenzdarlegung die Evidenzklassifikation des Oxford Centre for Evidence Based Medicine. Die Empfehlungen werden im Folgenden entsprechend der Konsistenz der Studienergebnisse, des Nutzen-Risiko-Verhältnisses, der Patientenpräferenzen und der klinischen Relevanz der Studienendpunkte und Effektstärken von der Leitliniengruppe graduiert. Zusätzlich zur Suche nach Primärpublikationen wird eine systematische Leitlinienrecherche durchgeführt. Diese wurde für den gleichen Suchzeitraum in der Leitliniendatenbank Guidelines International Network (GIN) durchgeführt. Unterschiedliche fachspezifische Ansichten zur Diagnostik, Therapie und Nachsorge des Mammakarzinoms zwischen den Experten werden durch formale Konsensusverfahren berücksichtigt. Hier werden in mehreren Stufen Techniken des Nominalen Gruppenprozesses (NGP), Delphi-Technik und formale Konsensuskonferenzen eingesetzt. Neben studiengestützen Empfehlungen mit unterschiedlich gewichteten Graden werden somit auch Statements als Resultat der strukturierten Konsensusfindung im Sinne einer „good clinical practice“ durch die interdisziplinär zusammengesetzte Gruppe formuliert.

Neben einer Langversion als Buchpublikation sind Kurzversionen in deutscher und englischer Sprache in diversen Fachorganen in Planung. Zur Implementierung werden die Vollversionen auch im Internet unter www.awmf-leitlinien.de, www.krebsgesellschaft.de, www.g-i-n.net verfügbar sein. Die Empfehlungen dieser aktualisierten Version richten sich an alle Ärztinnen und Ärzte und alle Angehörigen von Berufsgruppen, die mit der Versorgung von Patientinnen mit Brustkrebs befasst sind (Gynäkologie, Allgemeinmedizin, Radioonkologie, Hämatoonkologie, Psychoonkologie, Physiotherapie, Pflege etc.) sowie an alle an Brustkrebs erkrankten Frauen und deren Angehörige.
Da der Aspekt des sogenannten „Informed und Shared Decision Making“ im Rahmen einer verbesserten Arzt-Patientenkommunikation gestärkt werden und innerhalb von Leitlinien vermehrt abgebildet sein soll, befinden sich derzeit auch spezielle aktualisierte, evidenzbasierte und unabhängige Patienteninformationen unter aktiver Beteiligung von Patientenorganisationen und Selbsthilfegruppen in Vorbereitung. Dadurch bietet die S3-Leitlinie eine sinnvolle Möglichkeit, die Expertise der Patienten in die Gesundheitsversorgung einzubinden und die gebündelte Information auch für diese Zielgruppe in geeigneter Form zugänglich zu machen und zu implementieren. Somit bieten sie auch der Patientin und deren Angehörigen eine qualitätsgesicherte Wissensvermittlung.

\section{Bibliografie}

DOI http://dx.doi.org/10.1055/s-0031-1271507

Senologie 2011; 8: 87-88

(c) Georg Thieme Verlag KG Stuttgart · New York · ISSN 1611-6453

Korrespondenzadressen

Prof. Dr. med. Rolf Kreienberg

Ärztlicher Direktor der

Universitätsfrauenklinik Ulm

Prittwitzstr. 43

89075 Ulm

rolf.kreienberg@uniklinik-ulm.de

PD Dr. med. Achim Wöckel

Universitätsfrauenklinik Ulm

Prittwitzstr. 43

$89075 \mathrm{Ulm}$

achim.woeckel@uniklinik-ulm.de 